\title{
ON CERTAIN NEW AND EXACT SOLUTIONS OF THE EMDEN-FOWLER EQUATION AND EMDEN EQUATION VIA INVARIANT VARIATIONAL PRINCIPLES AND GROUP INVARIANCE
}

\author{
O. P. BHUTANI ${ }^{1}$ and K. VIJAYAKUMAR ${ }^{2}$
}

(Received 12 September 1989; revised 13 March 1990.)

\begin{abstract}
After formulating the alternate potential principle for the nonlinear differential equation corresponding to the generalised Emden-Fowler equation, the invariance identities of Rund [14] involving the Lagrangian and the generators of the infinitesimal Lie group are used for writing down the first integrals of the said equation via the Noether theorem. Further, for physical realisable forms of the parameters involved and through repeated application of invariance under the transformation obtained, a number of exact solutions are arrived at both for the Emden-Fowler equation and classical Emden equations. A comparative study with Bluman-Cole and scale-invariant techniques reveals quite a number of remarkable features of the techniques used here.
\end{abstract}

\section{Introduction}

The importance of invariant variational principles and group-invariance techniques for solving nonlinear differential equations of many a physical and engineering system has already been highlighted in a number of recent publications. See for example Logan [10], Bhutani and Mital [2], Bhutani et al [3], Bluman and Cole [1], Olver [12] and Bhutani et al [4-6].

The motivation for the present study had its origin in our attempt to carry over these techniques either singly or collectively, as the case may be, for obtaining the exact solutions of the Emden-Fowler equation and the classical Emden equation that have been listed in Section 2. More specifically,

\footnotetext{
${ }^{1}$ Department of Mathematics, Indian Institute of Technology, Hauz Khas, New Delhi-110016.

${ }^{2}$ Department of Mathematics, Panjab University, Chandigarh, India.

(C) Copyright Australian Mathematical Society 1991, Serial-fee code 0334-2700/91
} 
after formulating the alternate potential principle for the generalised EmdenFowler equation in Section 3, we have obtained, depending on the physical realisable forms of the parameters involved, three different first integrals of the Emden-Fowler equation via Noether's theorem. This is followed by the determination of exact solutions for the first-order equations via group invariance in Section 4. As a check to the efficacy of this technique, we have in Section 5, obtained exact solutions of the Emden-Fowler equation via a scaleinvariant transformation. In Section 6, we have examined the possibility of integrating the Emden-Fowler equation via the Bluman and Cole technique. It turns out that the condition for the integrability of the reduced form of the Emden-Fowler equation via the Bluman and Cole technique is the same as the one obtained in new dependent and independent variables. Under the condition, a class of new exact solutions is obtained. Finally, in Section 7 we have summed up the results of this study in the form of conclusions.

\section{Generalised Emden-Fowler equation and its applications}

The generalised Emden-Fowler equation is written in the following form

$$
y^{\prime \prime}+\alpha(t) y^{\prime}+\beta(t) y^{n}=0,
$$

where $\alpha(t)$ and $\beta(t)$ are arbitrary functions of $t$, and $n$ is a real number. Equation (2.1) is reduced to the classical Emden-Fowler equation [11]

$$
y^{\prime \prime}+\frac{k}{t} y^{\prime}-\beta_{0} t^{\omega} y^{n}=0,
$$

when $\alpha(t)=k / t, k$ is a constant parameter and $\beta(t)=-\beta_{0} t^{\omega}, \beta_{0}$ is another constant. Equation (2.2) has been the subject of study by Rosenau [13] for its solutions. Further, if $\beta_{0}=-1, \omega=0$ and $k=2$ in (2.2), we arrive at the Emden equation:

$$
y^{\prime \prime}+\frac{2}{t} y^{\prime}+y^{n}=0
$$

which arises in astrophysics [8]. Also, (2.2) can be considered as the generalised Thomas-Fermi equation [7]. When transformed to

$$
\vec{y}^{\prime}-\beta_{0}(k-1)^{(\omega+2 k) /(1-k)} \bar{t}^{(\omega+2 k) /(1-k)} \bar{y}^{n}=0
$$

under the transformation

$$
\bar{t}=t^{1-k} / k-1, \quad \bar{y}(\bar{t})=y(t),
$$

(2.4) assumes another form of Thomas-Fermi equation [9]. For the case $\omega=-7 / 2, n=3 / 2, \beta_{0}=1$ and $k=2$, we arrive at the usual ThomasFermi equation [9]

$$
\vec{y}^{\prime \prime}=(\bar{t})^{-1 / 2}(\bar{y})^{3 / 2} \text {. }
$$




\section{Invariant variational principle for the generalised Emden-Fowler equation and the first integrals}

For obtaining the invariant variational principle, we need to prove the existence and hence formulate a functional whose stationarity will yield the given differential equation. To this effect we follow our earlier works $[2,3]$ and write down the functional as follows:

$$
F[y]=\int_{\Omega}\left[\left(-\frac{1}{2} y^{\prime 2}+\frac{\beta(t)}{n+1} y^{n+1}\right) \exp \left(\int \alpha(t) d t\right)\right] d \Omega .
$$

Thus, the Lagrangian $L$, leading to (3.1) can be expressed as

$$
L=\left(-\frac{1}{2} y^{2}+\frac{\beta(t)}{n+1} y^{n+1}\right) \exp \left(\int \alpha(t) d t\right) .
$$

In order to prove the invariance of the fundamental integral $\left(\int L d t\right)$, we look for a one-parameter infinitesimal group of transformations of the form:

$$
\begin{aligned}
& t^{*}=t+\tau(t, y) \varepsilon+0\left(\varepsilon^{2}\right), \\
& y^{*}=y+\xi(t, y) \varepsilon+0\left(\varepsilon^{2}\right) .
\end{aligned}
$$

The necessary condition for the fundamental integral $\left(\int L d t\right)$ to be invariant under the transformation (3.3) as given by Rund [14] is

$$
\begin{aligned}
\frac{\partial L}{\partial t} \tau & +\frac{\partial L}{\partial y} \xi+\frac{\partial L}{\partial y^{\prime}}\left[\frac{\partial \xi}{\partial t}+\frac{\partial \xi}{\partial y} y^{\prime}-\frac{\partial \tau}{\partial t} y^{\prime}-\frac{\partial \tau}{\partial y} y^{\prime 2}\right] \\
& +L\left(\frac{\partial \tau}{\partial t}+\frac{\partial \tau}{\partial y} y^{\prime}\right)=0 .
\end{aligned}
$$

On substituting for $L$ and its derivatives in (3.4) and collecting in descending order the coefficients of various powers of $y^{\prime}$ and setting these coefficients equal to zero, we obtain the following system of partial differential equations

$$
\begin{gathered}
\frac{\partial \tau}{\partial y}=0, \quad \frac{\partial \xi}{\partial t}=0 \\
\frac{\partial \tau}{\partial t}-\dot{\gamma}(t) \tau-2 \frac{\partial \xi}{\partial y}=0, \\
y\left(\dot{\gamma}(t) \tau+\frac{\dot{\beta}}{\beta} \tau+\frac{\partial \tau}{\partial t}\right)+(n+1) \xi=0
\end{gathered}
$$

where

$$
\gamma(t)=\int \alpha(t) d t, \quad \dot{\gamma}=\frac{d}{d t}(\gamma), \quad \dot{\beta}=\frac{d}{d t}(\beta)
$$


On solving (3.5)-(3.6), we arrive at the following expressions for $\tau$ and $\xi$ :

$$
\begin{aligned}
& \tau(t)=e^{\gamma(t)}\left[a_{1} \int e^{-\gamma(t)} d t+a_{2}\right], \\
& \xi(y)=\frac{a_{1}}{2} y+a_{3},
\end{aligned}
$$

where $a_{2}$ and $a_{3}$ are constants of integration and $a_{1}$ is a separation constant. For a consistent solution to the system (3.5)-(3.7), equations (3.8)-(3.9) must satisfy (3.7). Consequently, the following possibilities arise:

(i) $a_{2}=0=a_{3}$, (ii) $a_{1}=0=a_{3}$ and (iii) $a_{3}=0$, where $\beta(t)$ and $\gamma(t)$ are related, corresponding to each case, through the relations

$$
\begin{gathered}
\left(\beta(t) e^{2 \gamma(t)}\right)^{2 / n+3} \int e^{-\gamma(t)} d t=\text { const } . \\
\beta(t) c^{2 \gamma(t)}=\text { const }, \text { for all } n, \\
\beta(t) e^{2 \gamma(t)}=\text { const, for } n=-3 .
\end{gathered}
$$

On making use of the invariance groups (3.8)-(3.9) in the following Noether's identity

$$
\left(-L+\frac{\partial L}{\partial y^{\prime}} y^{\prime}\right) \tau-\frac{\partial L}{\partial y^{\prime}} \xi=\text { const . , }
$$

we obtain, corresponding to the three different cases cited above, the following three different first integrals of (2.1):

$$
\begin{gathered}
\left(\frac{1}{2} y^{\prime 2}+\frac{\beta(t)}{n+1} y^{n+1}\right) e^{2 \gamma(t)} \int e^{-\gamma(t)} d t-\frac{1}{2} y y^{\prime} e^{\gamma(t)}=\text { const } . \\
\left(\frac{1}{2} y^{\prime 2}+\frac{\beta(t)}{n+1} y^{n+1}\right) e^{2 \gamma(t)}=\text { const } .
\end{gathered}
$$

and

$$
\frac{1}{2}\left(y^{\prime 2}-\beta(t) y^{-2}\right) e^{2 \gamma(t)}\left(a_{1} \int e^{-\gamma(t)} d t+a_{2}\right)-\frac{a_{1}}{2} e^{\gamma(t)} y y^{\prime}=\text { const } .
$$

Equations (3.14)-(3.16) are valid for arbitrary forms of $\alpha(t)$ and $\beta(t)$ that satisfy $(3.10)-(3.12)$. Corresponding to (2.2), the first integrals given by (3.14)-(3.16) and the respective conditions (3.10)-(3.12) assume, for $k=2$ the following forms:

$$
\begin{gathered}
\frac{t^{3}}{2} y^{\prime 2}+\frac{t^{2}}{2} y y^{\prime}-\frac{\beta_{0}}{n+1} t^{\omega+3} y^{n+1}=c_{1} \text {, a constant, } \quad 2 \omega=n-5, \\
\frac{t^{4}}{2} y^{\prime 2}-\frac{\beta_{0}}{n+1} y^{n+1}=c_{2}, \text { a constant, } \omega=-4 \text {, for all } n
\end{gathered}
$$


and

$$
\left(a_{2} t^{4}-a_{1} t^{3}\right) y^{\prime 2}-a_{1} t^{2} y y^{\prime}+\beta_{0} y^{-2}\left(a_{2}-a_{1} t^{-1}\right)=c_{3} \text {, a constant, } n=-3 .
$$

\section{Exact solution}

Herein, we deal with the first integrals listed in (3.17)-(3.19) for their exact solution via group invariance. Observing that (3.17) is further invariant under the transformation

$$
y=t^{-1 / 2} z
$$

that separates the variables in it, we get

$$
\pm \frac{2 \sqrt{n+1} d z}{\sqrt{4\left(c_{1}+2 \beta_{0} z^{n+1}\right)+(n+1) z^{2}}}=\frac{d t}{t},
$$

and $n \neq-1$. For $n=-3,0,1,(4.2)$ may be solved in terms of elementary functions. When $n=-7,-5,2,3,5,(4.2)$ can be solved and the solution can be expressed in terms of elliptic functions [13]. As a particular case, let us consider the situation when $c_{1}=0$. This leads to discussion of the following two cases:

CASE 1. $\beta_{0}>0$.

For the case under consideration, an exact solution to (4.2) can be expressed in the following form

$$
z=\left[\left(\frac{n+1}{8 \beta_{0}}\right)^{1 / 2} \frac{2(A t)^{(n-1) / 4}}{1-(A t)^{(n-1) / 2}}\right]^{2 /(n-1)},
$$

where $A$ is a constant of integration. On combining (4.1) and (4.2), we get

$$
y^{2}=\left(\frac{n+1}{2 \beta_{0}}\right)^{2 /(n-1)}\left[\frac{A}{\left(1-(A t)^{(n-1) / 2}\right)^{4 /(n-1)}}\right]
$$

Equation (4.4) represents a new exact solution to the Thomas-Fermi equation. It is interesting to point out that the exact solution given in (4.4), is not only new for the Thomas-Fermi model, but it is also valid for all $\beta_{0}>0, n>1$, $k=2$ and $2 \omega=n-5$. Further, (4.4) can be reduced to the solution of (2.4) under the transformation (2.5).

CASE 2. $\beta_{0}<0$. 
Let $\beta_{0}=-k_{1}^{2}, k_{1}>0$. Corresponding to this case, a solution to (4.2) can be expressed as

$$
z=\left[\left(\frac{n+1}{8 k_{1}^{2}}\right)^{1 / 2} \frac{2\left(A_{1} t\right)^{(n-1) / 4}}{1+\left(A_{1} t\right)^{(n-1) / 2}}\right]^{2 /(n-1)},
$$

where $A_{1}$ is another constant of integration. Combining (4.1) and (4.5), we get

$$
y^{2}=\left(\frac{n+1}{2 k_{1}^{2}}\right)^{2 /(n-1)}\left[\frac{A_{1}}{\left[1+\left(A_{1} t\right)^{(n-1) / 2}\right]^{4 /(n-1)}}\right],
$$

which represents an exact solution to the classical Emden-Fowler equations (2.2) for $k=2$. If we choose $n=5$, we arrive at the exact solution to the Emden equation (2.3), which coincides with the one reported in Murphy [11].

Corresponding to the first integral (3.18), one can write

$$
\pm \frac{d y}{\sqrt{c_{2}+\frac{2 \beta_{0}}{n+1} y^{n+1}}}=\frac{d t}{t^{2}} \text {. }
$$

As for (4.2), (4.7) may be solved in terms of elliptic functions, for particular values of $n$. however, for the case where $c_{2}=0$, we get

$$
y=\left(\sqrt{\frac{2 \beta_{0}}{n+1}}\left(\frac{1-n}{2}\right)\left(c-\frac{1}{t}\right)\right)^{2 /(1-n)},
$$

where $c$ is a constant of integration, a new solution to (2.2) for $\omega=-4$, $k=2$. The solution given in (4.8) is valid only when $(n+1)$ and $\beta_{0}$ have the same sign. It is worth mentioning that the first integral given in (3.19) corresponding to (2.2), for the case $n=-3$, is not solvable exactly.

\section{Invariant solutions of classical Emden-Fowler equation}

On using the scale-invariant transformation

$$
y \rightarrow a^{\gamma_{1}} y, \quad t \rightarrow a^{\gamma_{2}} t,
$$

where $\gamma_{1}$ and $\gamma_{2}$ are real constants and $a$ is another constant, we find that (2.2) is invariant under the infinitesimal transformations

$$
\begin{aligned}
& t^{*}=t+\varepsilon\left(\frac{1-n}{2+\omega}\right) t+0\left(\varepsilon^{2}\right), \\
& y^{*}=y+\varepsilon y+0\left(\varepsilon^{2}\right) .
\end{aligned}
$$


Consequently, (5.1) yields a transformation

$$
y=z(t) t^{(2+\omega) /(1-n)},
$$

which together with the following transformation

$$
t=e^{x}
$$

reduces (2.2) to the following autonomous ordinary differential equation:

$$
\begin{aligned}
z^{\prime \prime} & +\left(\frac{4+2 \omega+(1-n)(k-1)}{1-n}\right) z^{\prime} \\
& +\left(\frac{2+\omega}{1-n}\right)\left(\frac{\omega+n+k(1-n)}{1-n}\right) z-\beta_{0} z^{n}=0 .
\end{aligned}
$$

In order to solve (5.4), we consider the following possibilities:

(i) $z=$ const., (ii) $2 \omega+k+3=n(k-1)$ and (iii) $\omega+n+1+k(1-n)=0$. Thus, we have the following cases for detailed discussion:

CASE 1. $z=B$, a constant.

For the case under consideration, (5.4) yields

$$
B=\left(\frac{2+\omega}{\beta_{0}} \frac{n(1-k)+\omega+k+1}{(1-n)^{2}}\right)^{(1 /(n-1)} .
$$

On combining (5.2) and (5.3), we find that

$$
y=B t^{2+\omega /(1-n)},
$$

turns out to be a new exact solution of (2.2), which has not been reported in any form so far. As a particular case, if we choose $\omega=0, k=2, \beta_{0}=-1$, we get

$$
y=\left[\frac{2(n-3)}{(n-2)^{2}} t^{2}\right]^{1 /(1-n)} .
$$

Equation (5.7) represents a new solution to the Emden equation (2.3). Also, when $\beta_{0}=1, k=2, n=3 / 2, \omega=-7 / 2$, we arrive at the solution to the Thomas-Fermi equation (2.6) [9]

$$
\bar{y}=144(\bar{t})^{-3}
$$

CASE 2. $2 \omega+k+3=n(k-1)$.

For this case, (5.4) can be integrated to yield a first-order differential equation which for general value of $\theta_{1}$, the constant of integration involved, can 
yield solutions in terms of elliptic functions. However, for the case where $\theta_{1}=0$, a more general exact solution to the Emden-Fowler equation corresponding to $\beta_{0}<0$ Thomas-Fermi equation for $\beta_{0}>0$ can be expressed, respectively, as

$$
y=\frac{y_{0} t^{\alpha_{0}}}{\left(1+\beta_{1} t^{y_{0}}\right)^{\delta_{0}}}
$$

and

$$
y=\frac{y_{1} t^{\alpha_{0}}}{\left(1-\beta_{2} t^{\gamma_{0}}\right)^{\delta_{0}}}
$$

In (5.9) and (5.10), $y_{0}=\left(2 \beta_{1} l^{2}(n-1) / k_{0}^{2}\right)^{1 /(n-1)}, \beta_{0}=-k_{0}^{2}$. In (5.9), $y_{1}=\left(2 \beta_{2} l^{2}(n-1) / \beta_{0}\right)^{1 /(n-1)}, \beta_{1}=A_{2}^{l(n-1)}, \beta_{2}=A_{4}^{l(n-1)}, \alpha_{0}=2+\omega / 1-n+$ $l, \gamma_{0}=l(n-1), l=(k-1) / 2$ and $\delta_{0}=2 /(n-1)$. Further, $A_{2}$ and $A_{4}$ are constants of integration. Equations (5.9) and (5.10) reduce respectively to equations (4.6) and (4.4) for $l=1 / 2$. This shows that solutions represented in (5.9) and (5.10) contain (4.4) and (4.6) as a subclass of solutions for (2.2). Even though the forms of solutions (5.9) and (5.10) appear to be very simple, they have not been reported so far.

CASE 3. $\omega+n+1+k(1-n)=0$.

For this case, using the substitution

$$
W(z)=1 / z^{\prime}(x),
$$

(5.4) is reduced to Abel's type of equation of the first kind.

$$
W^{\prime}+(k-1) W^{2}+\beta_{0} z^{n} W^{3}=0 .
$$

For $k=1$, a solution to (5.4) can be expressed as

$$
z=\left(\frac{1-n}{2} \sqrt{\frac{2 \beta_{0}}{n+1}} x+C_{0}\right)^{2 /(1-n)},
$$

where $C_{0}$ is a constant of integration. On combining (5.2), (5.3) and (5.13), we get

$$
y=t^{2+\omega /(1-n)}\left[\frac{1-n}{2} \sqrt{\frac{2 \beta_{0}}{n+1}} \log t+C_{0}\right]^{2 /(1-n)} .
$$

Equation (5.14) represents a new exact solution to the Emden-Fowler equation (2.2) for $k=1$. 


\section{Reduction of classical Emden-Fowler equation to first-order and integrability condition}

On making use of Bluman and Cole's procedure [1], (2.2) can be reduced to the first-order equation (for details see Appendix)

$$
U U^{\prime}-\frac{2+\omega}{1-n} z U^{\prime}+\left(\frac{\omega+n+1}{1-n}+k\right) U-\beta_{0} z^{n}=0,
$$

where

$$
z=t^{-(2+\omega /(1-n))} y,
$$

and

$$
U(z)=t^{-(1+\omega+n) /(1-n)} y^{\prime} .
$$

From (A.7) we make use of the relation

$$
2 \omega=n(k-1)-(k+3)
$$

and integrating (6.1), we get

$$
\frac{U^{2}}{2}-\left(\frac{2+\omega}{1-n}\right)(z U)-\frac{\beta_{0} z^{n+1}}{n+1}=\theta_{1},
$$

where $\theta_{1}$ is a constant of integration. On combining (6.2) and (6.4), we get

$$
\frac{t^{2}}{2} y^{\prime 2}+\left(\frac{k-1}{2}\right) t y y^{\prime}-\frac{\beta_{0}}{n+1} t^{(k-1)(n-1) / 2} y^{n+1}=0 .
$$

In writing (6.5), the constant $\theta_{1}$ is assumed to be zero. Keeping in view the fact that $(6.5)$ is invariant under the transformation

$$
\left.\begin{array}{l}
W=t^{(k-1) / 2} y \\
V=t^{1-(1-k) / 2} y^{\prime}
\end{array}\right\}
$$

we can rewrite it as

$$
\frac{d t}{t}=\frac{d W}{G(W)-\frac{1-k}{2} W}
$$

where

$$
V=G(W)=\left(\frac{1-k}{2}\right) W \pm \sqrt{\frac{(k-1)^{2}}{2} W^{3}+\frac{2 \beta_{0}}{n+1} W^{n+1}}
$$

It is interesting to point out that on solving (6.7) for $W(t)$ and combining it with (6.6), we arrive, corresponding to the two choices for $\beta_{0} \gtrless 0$, 
at the expressions for $y$ that coincide, respectively, with (5.9) and (5.10). Further, the condition (6.3) under which (6.1) is integrable turns out to be the integrability condition for (2.2).

\section{Conclusion}

Following the invariant variational principles and group-invariant techniques, we have obtained quite a number of new or exact solutions to the generalised form of the Emden-Fowler equation and Emden equation. The most notable among them are the solutions (4.4) and (4.6) to (2.2) under the condition $2 \omega=n-5$. (c.f. Section 4) and (5.9) and (5.10) to (2.2) for all $\omega, n$ and $k$ satisfying the condition $2 \omega=n(k-1)-(k+3)$. Even though are solutions (5.9) and (5.10) are of a simple type, these solutions are not reported anywhere. Also, the solution (5.6) is new for all $\omega, n$ and $k$ for the Emden-Fowler equation (2.2), which, for a particular case, yields solutions of Emden equation (2.3) and Thomas-Fermi equation (2.6). Further, in Section 6, we have extended the study to obtain the generalised invariance group $(A-6)$ (see Appendix), which for a particular case yields the integrability condition for the Emden-Fowler equation (2.2).

\section{Appendix}

In order to apply Bluman and Cole's procedure to (2.1), we rewrite it as follows:

$$
y^{\prime \prime}=w\left(t, y, y^{\prime}\right)=-\alpha(t) y^{\prime}-\beta(t) y^{n},
$$

Keeping (A.1) invariant under the infinitesimal transformation

$$
\begin{aligned}
t^{*} & =t+\varepsilon \xi(t, y)+0\left(\varepsilon^{2}\right), \\
y^{*} & =y+\varepsilon \eta(t, y)+0\left(\varepsilon^{2}\right),
\end{aligned}
$$

then the invariant condition, as given in [1], is as follows:

$$
\begin{aligned}
\xi w_{t} & +\eta w_{y}+\left\{\eta_{t}+\left(\eta_{y}-\xi_{t}\right) y^{\prime}-\xi_{y} y^{\prime 2}\right\} w_{y^{\prime}} \\
& -\eta_{t t}-\left(2 \eta_{t y}-\xi_{t y}\right) y^{\prime}-\left(\eta_{y y}-2 \xi_{t y}\right) y^{\prime 2}+\xi_{y y} y^{\prime 3} \\
& -\left(\eta_{y}-2 \xi_{t}-3 \xi_{y} y^{\prime}\right)_{w}=0 .
\end{aligned}
$$

On making use of (A.1) in (A.3) and collecting the coefficients of various powers of derivatives of $y$ and equating them to zero, we arrive at the following 
system of partial differential equations involving $\xi, \eta$

$$
\begin{gathered}
\xi_{y y}=0 \\
-2 \alpha(t) \xi_{y}-\eta_{y y}+2 \xi_{t y}=0 \\
-\alpha^{\prime}(t) \xi-\alpha(t) \xi_{t}-2 \eta_{t y}+\xi_{t t}-3 \beta(t) y^{n} \xi_{y}=0, \\
-\beta^{\prime}(t) y^{n} \xi-n \beta(t) y^{n-1} \eta-\alpha(t) \eta_{t}+\eta_{t t}+\beta(t) y^{n}\left(\eta_{y}-2 \xi_{t}\right)=0 .
\end{gathered}
$$

The general solution to the system (A.4) is given as

$$
\begin{aligned}
& \xi=e^{\int \alpha(t) d t}\left[-2 \int\left(c_{1} \int e^{\int \alpha(t) d t} d t+c_{2}\right) e^{-\int \alpha(t) d t} d t\right]+c_{3} e^{\int \alpha(t) d t}, \\
& \eta=\left(c_{1} \int e^{\int \alpha(t) d t} d t+c_{2}\right) y
\end{aligned}
$$

where $c_{1}, c_{2}$ and $c_{3}$ are arbitrary constants. Making use of the choices of the arbitrary constants, we arrive at different possibilities of obtaining symmetries that keeps (A.1) invariant. When applying (A.5) to (2.2), with the corresponding choices of $\alpha(t)$ and $\beta(t)$ given therein, we obtain

$$
\begin{aligned}
& \xi=\frac{c_{1}}{k+1} t^{k+2}+\frac{2 c_{2} t}{1-k}+c_{3} t^{k}, \\
& \eta=\left(\frac{c_{1}}{k+1} t^{k+1}+c_{2}\right) y .
\end{aligned}
$$

In (A.6), if we make $c_{1}=c_{3}=0$, then we arrive at the following forms of $\xi$ and $\eta$ :

$$
\xi=\frac{2 c_{2}}{1-k} t, \quad \eta=c_{2} y \quad \text { and } \quad 2 \omega=n(k-1)-(k+3) .
$$

From (A.7), we write the Lagrange characteristic equation

$$
\frac{d t(1-k)}{2 c_{2} t}=\frac{d y}{c_{2} y}=\frac{d y^{\prime}\left(1-\frac{2}{1-k}\right)}{c_{2} y^{\prime}} .
$$

Solving (A.8), we find that

$$
\begin{aligned}
z & =t^{-(\omega+2 /(1-n)} y^{\prime} \\
U(z) & =t^{-(\omega+1+n) /(1-n)} y^{\prime}
\end{aligned}
$$

\section{References}

[1] G. W. Bluman and J. D. Cole, Similarity methods for differential equations (SpringerVerlag, 1974).

[2] O. P. Bhutani and P. Mital, "On the first integral of the nonlinear shallow-membrane equation via Noether's theorem", Int. J. Engng. Sci. 23 (1985) 353-357. 
[3] O. P. Bhutani, G. Chandrasekaran and P. Mital, "On the existence, formulation and invariance of variational principles for the differential equations of Nuclear engineering system and their exact solutions", Int. J. Engng. Sci. 26 (1988) 243-248.

[4] O. P. Bhutani and P. Mital, J. Meteor. Soc. Japan, Ser. II 64 (1986) 593-598.

[5] O. P. Bhutani, K. Vijayakumar, P. Mital and G. Chandrasekharan, "On invariant solutions of the generalised Korteweg-de-Vries equation in a variable medium-I", Int. J. Engng. Sci. 27 (1989) 921-929.

[6] O. P.Bhutani, K. Vijayakumar, P. Mital and G. Chandrasekaran, "On invariant solutions of the generalised Korteweg-de Vries-Burger type equations-II", Int. J. Engng. Sci. 27 (1989) 931-941.

[7] C. Y. Chan and Y. C. Hon, "A constructive solution for a generalised Thomas-Fermi theory of ionised atoms”, Quart. J. Appl. Math. 45 (1987) 591-599.

[8] S. Chandrasekhar, Principles of stellar dynamics (University of Chicago Press, Chicago, 1942).

[9] E. Kamke, Differential gleichungen losungs method en und losungen (Chelsa Publishing Company, 1948).

[10] J. D. Logan, Invariant variational principles (Academic Press, 1977).

[11] G. M. Murphy, Ordinary differential equations and their solutions (D. Van Nostrand Company Inc., 1960).

[12] P. J. Olver, Applications of Lie Groups to differential equations (Springer-Verlag, 1986).

[13] P. Rosenau, "A note on integration of the Emden-Fowler equation", Int. J. Nonlinear Mechanics, 19 (1984) 303-308.

[14] H. Rund, Hamilton-Jacobi theory in the calculus of variations (Princeton, New Jersey, 1966). 